\title{
Clinical features and genetic diagnosis of hereditary spinocerebellar ataxia 3
}

\author{
YAOGUANG WANG ${ }^{1 *}$, XIAOKAI YANG ${ }^{1 *}$, WEIDE MA ${ }^{2}$, JINXIN LI $^{2}$, QINGYUAN ZHANG $^{1}$, \\ SHUQI XIA ${ }^{2}$, HAI WANG ${ }^{2}$, CHENGHUI ZHANG ${ }^{2}$, XIAOMIN XU ${ }^{2}$ and JIAYONG ZHENG ${ }^{2}$
}

\begin{abstract}
Departments of ${ }^{1}$ Neurology and ${ }^{2}$ Gynecology and Obstetrics, Wenzhou City Key Laboratory of Gynecology and Obstetrics, Wenzhou People's Hospital, Wenzhou Maternal and Child Health Care Hospital, The Third Clinical Institute Affiliated to Wenzhou Medical University, Wenzhou, Zhejiang 325000, P.R. China
\end{abstract}

Received October 4, 2015; Accepted July 14, 2016

DOI: $10.3892 / \mathrm{mmr} .2016 .5707$

\begin{abstract}
Spinocerebellar ataxia type 3 (SCA3) is a rare inherited autosomal dominant progressive neurological disorder, which results from a CAG-repeat expansion in the gene encoding the deubiquitinating enzyme, ataxin-3. At present, no effective treatment is available for this fatal disorder; however, certain studies have suggested that reducing the levels of mutant ataxin-3 protein may reverse or halt the progression of disease in patients with SCA3. In the present study, clinical examinations were performed on a patient with SCA3 who exhibited disease features including coughing, expectoration and was bedridden with mobility limitation. CAG repetitions at SCA-associated genes were detected in the patient's family by performing standard polymerase chain reaction $(\mathrm{PCR})$ and triple-repeat primed PCR. The numbers of CAG-repeats within the two alleles of the gene of interest in the patient were 15 and 78. Notably, the patient's brother, who harbored 76 CAG-repeats in one allele of the gene of interest, did not exhibit severe disease symptoms. These results suggest that the number of CAG-repeats is a critical for determination of SCA3 disease severity and time of onset. In addition, the defined phenotypic characteristics of the patient in the present study provide useful insight for more accurate clinical diagnosis and genotyping of future patients.
\end{abstract}

Correspondence to: Dr Jiayong Zheng, Department of Gynecology and Obstetrics, Wenzhou City Key Laboratory of Gynecology and Obstetrics, Wenzhou People's Hospital, Wenzhou Maternal and Child Health Care Hospital, The Third Clinical Institute Affiliated to Wenzhou Medical University, 57 Canghou Road, Wenzhou, Zhejiang 325000, P.R. China

E-mail: zhengjiayongwz@foxmail.com

*Contributed equally

Key words: spinocerebellar ataxia type 3, genetic diagnosis, mutation

\section{Introduction}

Spinocerebellar ataxia (SCA) is a progressive, degenerative, genetic and neurodegenerative disease with multiple types, and currently no known effective treatment or cure (1). More than 60 different types of SCA that have been identified to date, which are diagnosed via autopsy (2). SCA3 is an autosomal dominant neurodegenerative disorder with numerous clinical features, including ataxia, ophthalmoplegia, pyramidal signs, basal ganglia symptoms and peripheral neuropathy (1). The causative gene, ataxin 3 (ATXN3) has been mapped to chromosome 14q32.1 (2). Nakano et al (2) first reported SCA3 in 1972 in the Machado family, who were Portuguese immigrants living in Massachusetts. In 1976, Rosenberg et al (3) identified SCA3 in the Joseph family. Subsequently, it was demonstrated that SCA3 is the result of expansion of CAG trinucleotide-repeats in the ATXN3 gene. Sequences of healthy individuals contain 14-40 CAG-repeats; however, sequences of patients with SCA3 contain 72-86 CAG-repeats (4). The numbers of expanded repeats vary between generations, resulting in significant phenotypic variations; patients with increased numbers of triplet-repeats suffer from a greater disease severity and earlier onset (5). The triplet repeat primed polymerase chain reaction (TP-PCR) method was developed to screen for expanded alleles.

In 1997, Zhou et al (4) confirmed the expansion of CAG-repeats in the ATXN3 gene in Chinese patients with SCA3. However, to date, few cases of SCA3 have been reported in the Chinese population. In the present study, a Chinese family with SCA3 was identified, and the genetic and clinical characteristics of these patients reported. The results of the present study provide insight that may improve the accuracy of the clinical diagnosis for SCA3.

\section{Materials and methods}

Patients and diagnoses. Blood samples from the patient and his siblings were collected and treated with an anticoagulant, ethylenediaminetetraacetic acid. The diagnosis of SCA was based on established criteria (6). Interviews with the patient and his family were performed to obtain information on family history. The study was approved by the ethics committee of 
Wenzhou People's Hospital (Wenzhou, China). Informed consent was obtained from all subjects prior to blood sample collection. Clinical assessments, including regular neurological tests, magnetic resonance imaging (MRI) and ocular examinations were conducted at the Wenzhou People's Hospital.

Sample analysis and PCR. DNA was isolated from peripheral blood lymphocytes using the QIAamp DNA Blood Mini kit (Qiagen GmbH, Hilden, Germany). SCA1, SCA2, SCA3, SCA6, SCA7 and dentatorubropallidoluysian atrophy (DRPLA) loci were amplified by PCR using 5'-carboxyfluorescein-labeled primers and AmpliTaq Gold ${ }^{\circledR}$ DNA polymerase (catalog no. N8080247; Applied Biosystems; Thermo Fisher Scientific, Inc., Waltham, MA, USA) as previously described (1,7-10). PCR products were analyzed with capillary electrophoresis according to the previous study (4) using the ABI 3130xl system (Applied Biosystems; Thermo Fisher Scientific, Inc.). Standard PCR was performed with a reaction volume of $50 \mu \mathrm{l}$, containing $100 \mathrm{ng}$ genomic DNA, $2 \mathrm{pM}$ each primers and $25 \mu 1$ 2X Taq PCR Master Mix (Biotake GmbH, Glashütten, Germany). The PCR process was performed using a thermal cycler platform (Applied Biosystems; Thermo Fisher Scientific, Inc.). TP PCR assay was performed in a reaction volume of $25 \mu \mathrm{l}$, which contained $200 \mathrm{ng}$ DNA, $1.5 \mathrm{mmol} / \mathrm{l}$ $\mathrm{MgCl}_{2}, 10 \mathrm{mmol} / 1$ Tris, $50 \mathrm{mmol} / 1 \mathrm{KCl}, 0.8 \mu \mathrm{mol} / 1$ primers, $200 \mu \mathrm{mol} / 1 \mathrm{dNTPs}$ each and $2 \mathrm{U}$ Taq polymerase (Eppendorf AG; Hamburg, Germany).

\section{Results}

A case of SCA3 was diagnosed in a Chinese family by identification of CAG expansion at the SCA3 locus using PCR. The proband patient (III-3) was a 40-year-old male who presented with coughing and expectoration and was with bedridden with mobility limitation. The patient first noticed symptoms, including difficulty walking in a straight line and a tendency to fall, about 20 years previously. Five years prior to the present study, the patient began to experience severe neurological problems, including choking and dribbling while drinking. Disease symptoms worsened approximately one year ago, as the patient became incontinent and unable to feed himself. Three days prior to the present study, the patient became unable to walk and was experiencing coughing and expectoration. Among the other members of his family, the father of the patient died of SCA and his elder brother walked unsteadily. However, his mother and elder sister were healthy (Fig. 1).

Clinical examinations demonstrated that the patient was alert and fully oriented with a blood pressure of $110 / 60 \mathrm{mmHg}$ and a resting heart rate of $72 \mathrm{bpm}$. The patient exhibited neck abnormalities, dysarthria, and moderate dysmetria as assessed by the finger-to-nose and heel-knee-shin test. Pupils were symmetrical and sensitive to light with signs of nystagmus and restricted eye movement. In addition, the patient was suffering from dysphagia and amyotrophy of the tongue. Other physiological measurements of the patients were as follows: Glutamic-oxalacetic transaminease, $165 \mathrm{U} / 1$; creatine kinase, $2419 \mathrm{U} / 1$; and creatine kinase isoenzyme, 45 U/l. An MRI scan of the head identified brain, cerebellar and brain stem atrophy, typical of patients with SCA (Fig. 2).

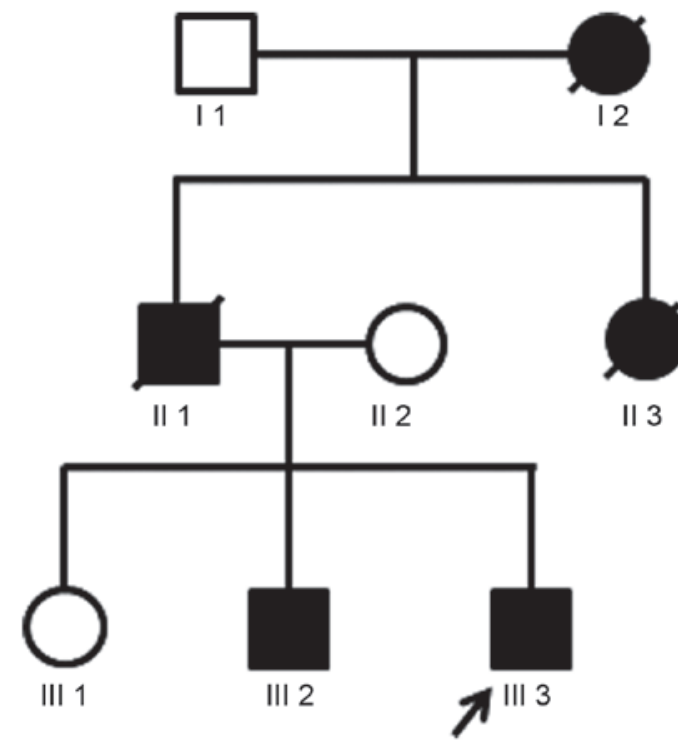

Figure 1. Spinocerebellar ataxia type 3 status and pedigree analysis of the selected family. Closed symbols indicate affected individuals; open symbols indicate unaffected subjects. A slash through the symbol indicates that the family member is deceased. The arrow indicates the proband.

The genotyping results of blood samples collected from family members are presented in Fig. 3. PCR amplification and capillary electrophoresis analysis identified a CAG-repeat length of 15 in the normal allele and an expanded 78 CAG-repeat in the patient III-3. A 76 CAG-repeat was identified in his brother III-2, while 20 CAG-repeats were identified in his sister III-1. The CAG-repeats within the SCA1, SCA2, SCA6, SCA7, SCA12 and DRPLA genes of the patient were within the normal range.

\section{Discussion}

SCA diseases have been classified into three groups by Harding et al (6), according to clinical features. These classification criteria remain the guidelines for genetic diagnosis of SCA. SCA3 belongs to type I autosomal dominant cerebellar ataxia (ADCA), which includes ataxia accompanied by optic atrophy, ophthalmoplegia, extrapyramidal signs, neuropathy and cognitive impairment. The subject of the present study demonstrated signs of slurred speech, moderate dysmetria as assessed by the finger-to-nose and heel-knee-shin test, dysphagia, amyotrophy of the tongue, symmetrical pupils, sensitivity to light and restrictive movement of eyes, which are typical of type I ADCA. However, symptoms overlapping the three types of SCA are frequently observed in patients. SCA3 results from mutations involving CAG trinucleotide-repeat expansions in the coding regions of the ATXN3 gene. Healthy individuals have a small and stable number of CAG-repeats (14-40). However, disease occurs when the CAG-repeats exceed a certain size (4). A feature of SCA resulting from CAG-repeat expansion is genetic anticipation, as the number of CAG-repeats typically increases with each subsequent generation. This increase in the numbers of CAG-repeats leads to a greater disease severity and earlier onset (5). While normal alleles are stably transmitted without modification, mutated alleles are unstable and further expansion of CAG-repeats may occur during transmission. However, the 


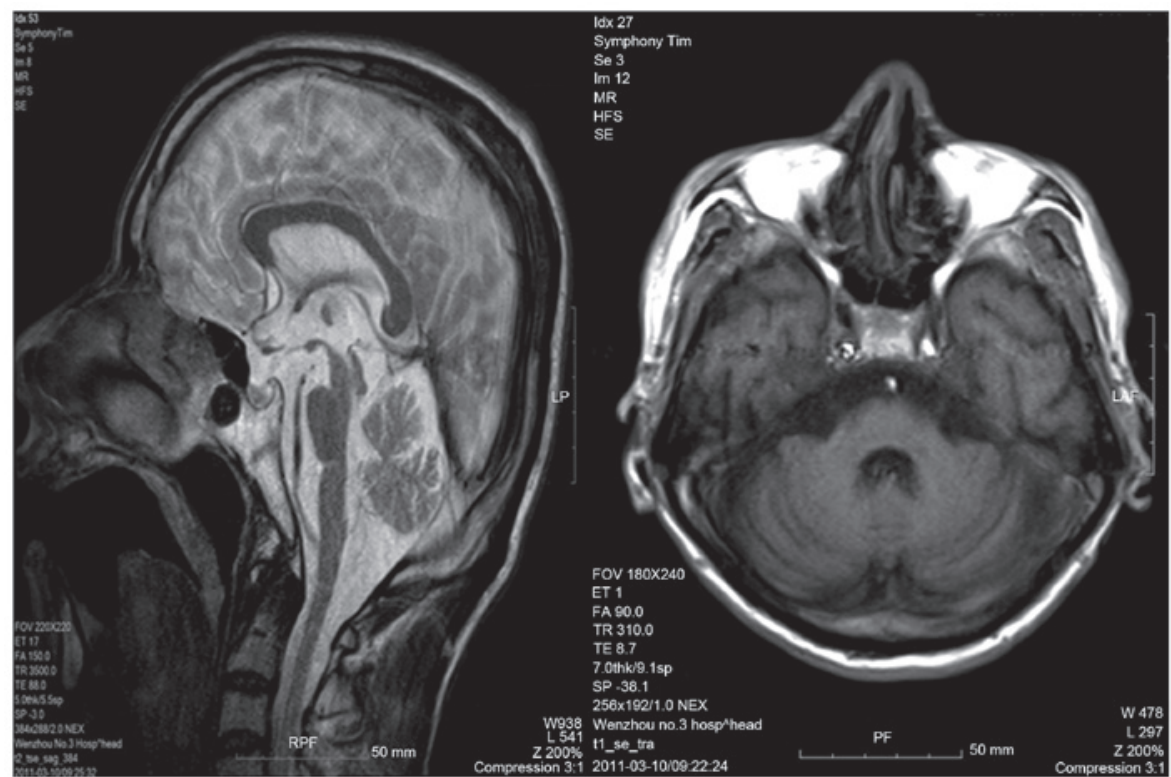

Figure 2. Head MRI of the proband patient. MRI demonstrated brain, cerebellar and brain stem atrophy, typical of SCA. MRI, magnetic resonance imaging.
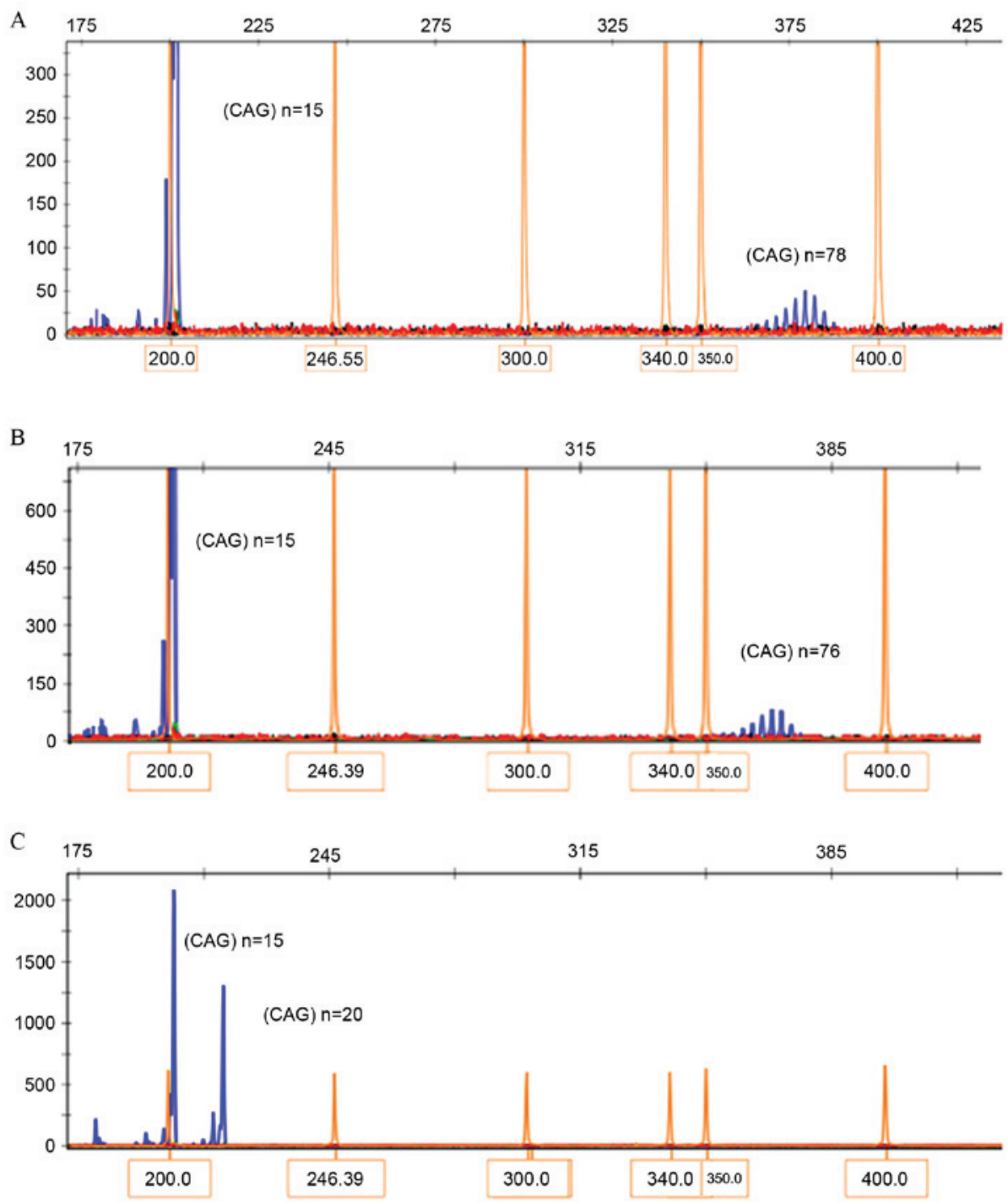

Figure 3. Spinocerebellar ataxia type 3 loci of the patient and selected family. (A) Numbers of CAG repeats in the patient alleles were 15 and 78 . (B) Numbers of CAG repeats in the alleles of the brother of the patient were 15 and 76. (C) Numbers of CAG repeats in the alleles of the sister of the patient sister were 15 and 20, the blue line indicate the CAG repeats. 
underlying mechanism by which CAG-repeat expansion occurs remains to be fully elucidated. Typically, disease severity correlates with the extent of CAG-repeat expansion: The greater the number of CAG-repeats, the greater the severity of the disease and the earlier its onset. In the present study, the patient harboring 78 CAG-repeats at the disease gene demonstrated an earlier onset of SCA3 compared with his brother, who had fewer CAG-repeats. These results are consistent with the observations of our previous study (11).

Due to somatic mosaicism, variable repeat sizes in different tissues of the same patient may influence disease onset and severity. ATXN3 exerts greater toxicity in the nucleus compared with the cytoplasm. Previous studies have demonstrated that ATXN3 interacts with various transcription regulators to modulate the cellular stress response, and directly regulates the ubiquitin proteasome system. CAG-repeat expansion in ATXN3 altered its affinity for other regulators, which may explain the pathogenesis of SCA3 $(12,13)$.

Short tandem repeat analysis based on capillary electrophoresis is a simple and reliable method for the detection of CAG-repeats. However, PCR using flanking primers only allows amplification up to 100 CAG-repeats; amplification of PCR templates over this size is unreliable. Triplet-repeat primed PCR (TP-PCR), by contrast, allows rapid identification of large pathogenetic CAG-repeats that may not be amplified using standard PCR (14). Therefore, TP-PCR was used to confirm the presence of large expansions in the present study when only one allele was identified by standard PCR (data not shown).

In conclusion, the present study reported the clinical symptoms and genetic characteristics of a Chinese family with SCA3. These observations provide insight into the clinical diagnosis and genetic typing of patients with SCA3, which may benefit future patients.

\section{Acknowledgements}

The authors would like to thank the patient and his family for their participation in the present study. The present study was supported by the Zhejiang Provincial Natural Science Foundation of China (grant no. Y13H040023; to Dr Jiayong Zheng) and the Wenzhou Science and Technology Foundation (grant no. Y20140408; awarded to Dr Yanhui Jin, The First Affiliated Hospital of Wenzhou Medical University, Wenzhou, China).

\section{References}

1. Kawaguchi Y, Okamoto T, Taniwaki M, Aizawa M, Inoue M, Katayama S, Kawakami H, Nakamura S, Nishimura M, Akiguchi I, et al: CAG expansions in a novel gene for Machado-Joseph disease at chromosome 14q32.1. Nat Genet 8: 221-228, 1994.

2. Nakano KK, Dawson DM and Spence A: Machado disease. A hereditary ataxia in Portuguese emigrants to Massachusetts. Neurology 22: 49-55, 1972.

3. Rosenberg RN, Nyhan WL, Bay C and Shore P: Autosomal dominant striatonigral degeneration. A clinical, pathologic, and biochemical study of a new genetic disorder. Neurology 26: 703-714, 1976.

4. Zhou YX, Takiyama Y, Igarashi S, Li YF, Zhou BY, Gui DC, Endo K, Tanaka H, Chen ZH, Zhou LS, et al: Machado-Joseph disease in four Chinese pedigrees: Molecular analysis of 15 patients including two juvenile cases and clinical correlations. Neurology 48: 482-485, 1997.

5. Maciel P, Gaspar C, DeStefano AL, Silveira I, Coutinho P, Radvany J, Dawson DM, Sudarsky L, Guimarães J, Loureiro JE, et al: Correlation between CAG repeat length and clinical features in Machado-Joseph disease. Am J Hum Genet 57: 54-61, 1995.

6. Harding AE: Clinical features and classification of inherited ataxias. Adv Neurol 61: 1-14, 1993

7. Zhuchenko O, Bailey J, Bonnen P, Ashizawa T, Stockton DW, Amos C, Dobyns WB, Subramony SH, Zoghbi HY and Lee CC: Autosomal dominant cerebellar ataxia (SCA6) associated with small polyglutamine expansions in the alpha 1A-voltage-dependent calcium channel. Nat Genet 15: 62-69, 1997.

8. Stevanin G, Giunti P, Belal GD, Dürr A, Ruberg M, Wood N and Brice A: De novo expansion of intermediate alleles in spinocerebellar ataxia 7. Hum Mol Genet 7: 1809-1813, 1998.

9. Sanpei K, Takano H, Igarashi S, Sato T, Oyake M, Sasaki H, Wakisaka A, Tashiro K, Ishida Y, Ikeuchi T, et al: Identification of the spinocerebellar ataxia type 2 gene using a direct identification of repeat expansion and cloning technique, DIRECT. Nat Genet 14: 277-284, 1996.

10. Koide R, Ikeuchi T, Onodera O, Tanaka H, Igarashi S, Endo K, Takahashi H, Kondo R, Ishikawa A, Hayashi T, et al: Unstable expansion of CAG repeat in hereditary dentatorubral-pallidoluysian atrophy (DRPLA). Nat Genet 6: 9-13, 1994.

11. Lin Y, Zheng JY, Jin YH, Xie YC and Jin ZB: Trinucleotide expansions in the SCA7 gene in a large family with spinocerebellar ataxia and craniocervical dystonia. Neurosci Lett 434: 230-233, 2008

12. Todi SV, Scaglione KM, Blount JR, Basrur V, Conlon KP, Pastore A, Elenitoba-Johnson K and Paulson HL: Activity and cellular functions of the deubiquitinating enzyme and polyglutamine disease protein ataxin-3 are regulated by ubiquitination at lysine 117. J Biol Chem 285: 39303-39313, 2010.

13. Reina CP, Zhong X and Pittman RN: Proteotoxic stress increases nuclear localization of ataxin-3. Hum Mol Genet 19: 235-249, 2010

14. Warner JP, Barron LH, Goudie D, Kelly K, Dow D, Fitzpatrick DR and Brock DJ: A general method for the detection of large CAG repeat expansions by fluorescent PCR. J Med Genet 33: 1022-1026, 1996. 